\title{
Quality of kids' screen time matters as much as quantity
}

- Cite as: CMAJ 2019 June 24;191:E721. doi: 10.1503/cmaj.109-5767

Posted on cmajnews.com on June 6, 2019.

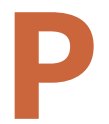

arents often worry that their kids are spending too much time in front of screens. A new statement from the Canadian Paediatric Society (CPS) warns that kids shouldn't be left to their devices, but the quality of screen time matters as much as the quantity.

"It's more useful to focus on how screens are being used and ensuring that screen use isn't interfering with other aspects of their lives," said Dr. Michelle Ponti, chair of the CPS Digital Health Task Force. "We need to prioritize school activities, physical activity, sleep, and social activities like family meals before reaching for a device."

The CPS recommends doctors counsel parents to be present and engaged when their children are using screens, and to encourage "meaningful" screen activities that are educational, active and social over those that are passive and solitary. Parents should also model healthy digital habits, such as turning off screens while driving and at least an hour before bed.

The statement doesn't recommend specific limits for school-aged kids and teens. According to CPS, the risks and benefits of digital media are nuanced, and may differ from one child or activity to another.

For example, watching "high-quality, immersive programming" as a family can be educational and promote social skills in children aged 5-12. But kids that age are also more likely to suffer negative outcomes from heavy screen use, including depressive symptoms.

While teens can reap cognitive and psychosocial benefits from using digital technology 2-4 hours a day, research links both zero use and excessive use to negative effects. Studies also connect screen use with sleep problems, decreased physical activity and weight gain.

It's estimated teens in Ontario and Alberta spend more than 7.5 hours per day on various screens. One in five spend more than five hours per day on social media alone. However, this doesn't necessarily indicate problematic screen use.

According to CPS, signs of problematic use include complaining about being bored or unhappy without access to devices, negative emotions after using devices, challenge behavior over screen limits and screen use that interferes with daily routines. The odd squabble over screen time shouldn't raise alarm, though. According to the statement, "occasional occurrence of these signs may be expected."

Lauren Vogel, CMAJ 